\title{
Author Correction: A proofreading-impaired herpesvirus generates populations with quasispecies-like structure
}

Jakob Trimpert, Nicole Groenke, Dusan Kunec, Kathrin Eschke, Shulin He, Dino P. McMahon and Nikolaus Osterrieder (D)

Correction to: Nature Microbiology https://doi.org/10.1038/s41564-019-0547-x, published online 2 September 2019.

In the version of this Letter originally published, in the sentence beginning "Conserved residues within the...", the values of the residual enzymatic activity were in the wrong order as ' 20 and $85 \%$ '; instead the sentence should have read "Conserved residues within the ExoIII domain were exchanged in the Y547S and Y567F mutants, which leaves a residual enzymatic activity of approximately 85 and $20 \%$, respectively (Fig. $1 \mathrm{~b}$ and Supplementary Table 1)." This has now been corrected. 